\title{
COMMUNICATION CHIMIQUE ET MIGRATION REPRODUCTRICE CHEZ LES SALMONIDES
}

Ph. SAGLIO

Laboratoire de Physiologie des Poissons, I.N.R.A.

78350 JOUY EN JOSAS. France

PLAN

A - Position du problème

B - Démonstration de la validité de l'hypothèse olfactive

1. Expériences de transplantation et d'imprégnation artificielle

2. Interactions phéromonales

C - Conclusion

Références bibliographiques.

\section{A - POSITION DU PROBLEME}

Parmi les Salmonidés, de nombreuses espèces, en particulier celles appartenant aux genres Salmo et Oncorhynchus, se caractérisent par leur capacité à retourner frayer dans le cours d'eau natal après un séjour en mer dont la durée varie selon celles-ci. 
Ce phénomène fascinant, connu dans son ensemble depuis fort longtemps. ayant déjà fait l'objet de nombreuses revues de qualité (voir en particulier HASLER, 1971; HARDEN JONES, 1968; STASKO, 1971; FONTAINE, 1975). nous ne traiterons ici que de la phase reproductrice de la migration ou * homing * (des eaux côtières proches de l'estuaire aux sites de fraie). dont la nature sensorielle (et p!us particulièrement olfactive) du determinisme ne fait plus aucun doute aujourd'hui.

Si l'étude de la phase océanique reste, pour des raisons évidentes de difficultés expérimentales, encore une énigme où les suppositions sont rares, il n'en est pas de même de celle du * homing * pour lequel les premières hypothèses connues, telle l'* hypothese olfactive * de BUCKLAND (1880) (citè par JONES, 1959) remontent à près d'un siècle.

Outre cette dernière, d'autres hypothèses accordant une place essentielle aux caractéristiques physico-chimiques de l'eau ont été formulées. Parmi celles-ci, on peut retenir celle de WARD (1921 a, b, 1939) et de HOAR (1958) selon laquelle la perception par le saumon de gradients thermiques constituerait le principal facteur régissant sa remontée jusqu'aux sites de fraie et celle de POWERS (1939), de POWERS et CLARK (1943) et de COLLINS (1952) pour lesquels le parametre responsable serait la tension en gaz carbonique de l'eau.

Bien que ces paramètres environnementaux puissent effectivement jouer un rô'e dans la remontée migratoire des Salmonidés, du fait de leur action sur le rhéotropisme (NORTHCOTE, 1962) et sur l'orientation de ces poissons, ils restent insuffisants, selon HASLER (1971), pour rendre compte de la remarquable spécificité du choix effectué par le Saumon vis-à-vis de son affluent d'origine.

\section{B - DEMONSTRATION DE LA VALIDITE DE L'HYPOTHESE OLFACTIVE}

\section{Expériences de transplantation et d'impregnation artificielle}

Parmi les quelques auteurs s'étant ralliès au début de ce siècle à l'hypothèse olfactive de BUCKLAND (CHIDESTER, 1924; CRAIGIE, 1926; KYLE, 1926 ; SCHEURING, 1930; WHITE, 1934). seuls CRAIGIE et, à un degré plus élevé, WHITE, purent lui apporter un soutien expérimental.

Après implantation de juvéniles de saumon atlantique, Salmo salar, dans une rivière de Nouvelle Ecosse connue comme ne possédant pas de représentant de cette espèce. WHITE put y constater, peu après un an, une importante remontée d'adultes.

Bien que l'origine de la fraie utilisee dans cette experience n'ait malheureusement pu être définie, cette étude eut le mérite d'une part de mettre l'accent sur la probabilité d'une reconnaissance intraspécifique d'origine sensorielle garantissant le phénomène du *homing " et, d'autre part, de proposer un protocole expérimental de terrain (méthode de transplantation) susceptible, moyennant certains ajustements, d'en contrôler les principaux aspecs éco-éthologiques.

Les diffèrentes études de transpiantation d'œufs et d'alevins de saumon atlantique ou de cohos (Oncorhynchus kisutch) (CARLIN, 1959, 1968; DONALDSON et ALLEN, 1957 ; PECK, 1970; JENSEN et DUNCAN, 1971) ont montré que 
la connaissance des caractéristiques du cours d'eau repose en partie sur un processus d' engrammation mnésique " réalisé au moment ou le juvénile commence sa migration aval.

L'hypothèse olfactive proposée en 1951 par HASLER et WISBY implique que : poisson ;

- Le cours d'eau possède une odeur spécifique perceptible par le

- Le poisson soit capable de discriminer entre les odeurs de différents cours d'eau ;

- Le poisson puisse conserver "l'engramme olfactif" caractéristique (existence d'une mémoire à long terme à support olfactif).

Afin de déterminer l'importance du róe joué par l'olfaction dans le choix de la rivière et le retour au site de fraie, ces auteurs proposèrent d'imprégner ("imprinting") les poissons, de l'œuf au stade "smolt", à un composé chimique n'existant pas dans la nature, ne possédant pas a priori d'action attractive ou répulsive, très soluble dans l'eau et perceptible à faible concentration.

Douze à dix huit mois après le lâcher initial des smolts marqués, le composé serait introduit dans la rivière expérimentale et l'efficacité de l'imprégnation o'factive pourrait être apprécièe grãce au nombre de poissons marqués capturés.

La "morpho!ine" (P.M. = 87) perçue à une concentration aussi faible que $5.10^{-5} \mathrm{mg} / \mathrm{l}$ fut le composé retenu (WISBY, 1952).

De nombreuses expériences d'imprégnation artificielle à ce composè réalisées ces dernières années aux Etats Unis et au Canada sur le Coho et la Truite arc-en-ciel (Salmo gairdner) (MADISON et al., 1978; SCHOLZ et al., 1975 ; COOPER et SCHOLZ, 1976 ; COOPER et al., 1976) attestent de l'efficacité de cette mèthode capable d'augmenter, par rapport à la seule imprégnation naturelle, les taux de retour des adultes dans un endroit donné.

Toutefois, d'après les enregistrements électro-physiologiques réalisés par HARA (1974), la stimulation du bulbe o'factif de la Truite arc-en-Ciel avec une solution de morpholine à $1 \%$ entraine une réponse différente de la réponse normale du bulbe olfactif.

Selon cet auteur, le mécanisme responsable de l'imprégnation à la morpholine n'est pas directement associé à la fonction olfactive. Ce qui remet en question la valeur de cette méthode, proposée initialement pour mettre en évidence l'existence d'une relation entre l'olfaction et le *homing", et inhibe la sensibilité olfactive à un acide aminé telle que la $L$ sérine.

Considerant ceci, on introduirait dans l'environnement de poissons expérimentaux (ou non) une substance synthétique dont on ignore la nature exacte de l'effet psychophysiologique, tout en risquant de perturber les capacités de perception olfactive à d'autres composés tels les acides aminés qui semblent jouer un rôle essentiel dans la vie des Salmonidés (voir à ce sujet les études d'IDLER et al., 1956 ; SUTTERLIN et SUTTERLIN, 1971 ; HARA, 1974 ; HARA et $a^{\prime}$, 1973).

Quoi qu'il en soit, les récents enregistrements électrophysiologiques réalisés sur le coho et la truite arc-en-ciel par COOPER et HASLER (1976) apportent, 
sans ambiquité, la preuve de la relation existant entre la stimulation à la morpholine chez des poissons imprégnés préalablement (à dose trés faible : $5.10^{-\bar{j}} \mathrm{mg} / \mathrm{l}$ ) ei l'apparition de réponses très nettes au niveau des bulbes olfactifs.

Selon ces mêmes auteurs, le rôle prédominant de l'olfaction dans le mécanisme de l'imprégnation artificielle est incontestable. Reprenant un de leurs arguments, si les rèponses étaient effectivement dues au $\mathrm{pH}$ élevè ou à un effet irritant (HARA et MAC DONALD, 1975) des solutions de morpholine, on ne serait plus en droit d'attendre de différence au niveau des réponses recueillies entre les poissons imprégnés et les poissons témoins.

Or, ces auteurs relèvent de très significatives différences entre les deux lots étudiés. D'autre part COOPER et HASLER ne constatent pas d'effet inhibiteur de la morpholine à $1 \%$ sur la réponse à un acide aminé tel que la L. méthionine et ajoutent que cette dernière concentration ne peut être considérée comme référentielle, les expériences d'imprégnation étant réalisées avec des doses de l'ordre de $10^{-5} \mathrm{mg} / \mathrm{l}$.

Toujours est-il qu'à ces études d'imprégnation artificielle, de finalité discutée bien qu'efficace dans les faits, se joignent un grand nombre d'expériences de transplantation (voir revue établie par STASKO, 1971), consistant à pratiquer simultanément des lâchers-recaptures de poissons marqués témoins et de poissons marqués porteurs de lésions olfactives ou (et) visuelles, pour démontrer à la fois la possibilité d'implantation d'une nouvelle population de salmonidés dans une rivière donnée et la prééminence de l'olfaction dans le phènomène du "homing

\section{Interactions phéromonales}

Malgré l'expérience prometteuse de WHITE et la conjecture de CHIDESTER (1924) qui proposait dix ans plus tôt d'expliquer le "homing" du saumon atlantique par attraction exercée par les jeunes sur les adultes, l'hypothèse phéromonale est restée longtemps inexplorée.

Jusqu'en 1971, date à laquelle NORDENG put montrer que les adultes des populations migratrices de l'Omble chevalier (Salvelinus alpinus) étaient attirés par les jeunes de la même espéce, la plupart des auteurs partisans d'une hypothèse chèmosensorielle rattachaient globalement avec HASLER (1956a, 1966) le homing à la perception de substances odorantes d'origine organique et minérale caractéristiques de l'affluent original.

En 1973, HOGLUND et ASTRAND, utilisant un type d'aquaolfactometre à choix multiples ("fluvarium ") (fig. 1) mettaient en évidence chez les juvéniles de l'Omble chevalier une attraction intraspécifique d'origine olfactive.

Les enregistrements électrophysiologiques réalisés par DOVING et al (1973) sur cette même espèce confirmaient les résultats de ces tests comportementaux.

Les auteurs recueillaient des reponses très nettes au niveau des bulbes olfactifs lors de la stimulation de l'épithélium olfactif avec une eau chargée de l'odeur de conspécifiques.

Après réalisation et test de différents extraits (mucus, sang, muscle, urine, laitance et granulés alimentaires), ces mêmes auteurs montrèrent que le mucus pouvait constituer le principal agent responsable des réponses observées. 
EXPERIENCE I

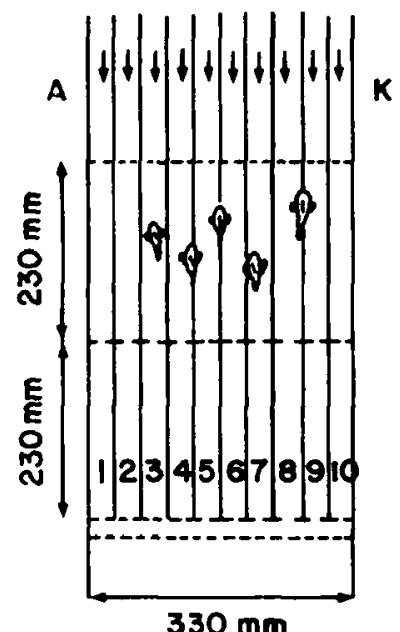

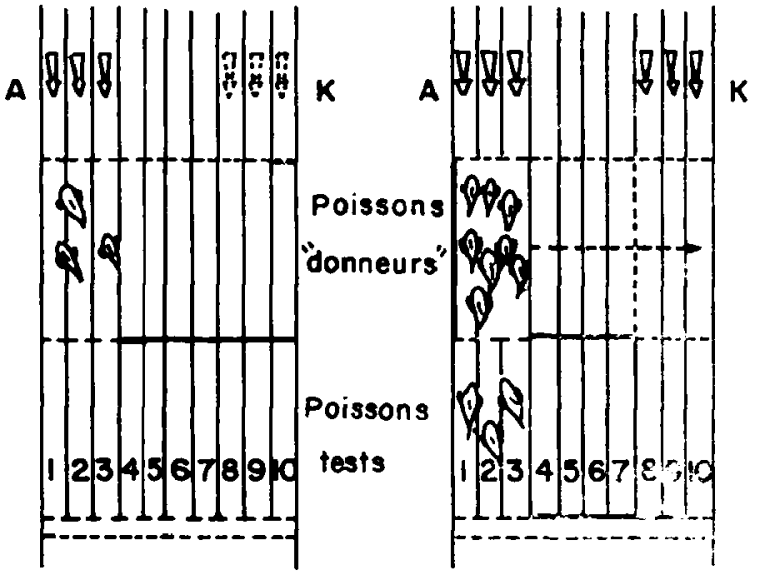

Figure 1 : Aquaolfactomètre à choix multiple ("Fluvarium "). Principe de fonctionnement (d'après HOGLUND et ASTRAND, 1973)

Si l'on considere les nombreuses demonstrations de l'action effective de sécrétions épidermiques dans le phénomène social des Poissons, en particulier Cyprinidés et Siluridés (voir à ce sujet WREDE, 1932; GOZ, 1941; VON FRISCH, 1941b; PFEIFFER, 1962; TODD et al., 1967; RICHARDS, 1974) la probabilité d'existence d'un tel phénomène chez les Salmonidés n'a rien de surprenant. Dans des études électrophysiologiques complémentaires DOVING et al., (1974) ont pu évoquer des réponses différentes selon l'origine des populations (migratrices ou sédentaires) d'omble testées. Des études du même ordre réalisées par OSHIMA et al. (1969) sur une espèce de saumon du genre migrateur Oncorhynchus ( $O$. tsawytscha) confirment la remarquable spécificité de la reconnaissance olfactive phéromonale chez les Salmonidés. Ces auteurs enregistrèrent des réponses olfactives à de l'eau ne provenant pas de la rivière spécifique, mais ayant contenu pendant toute une nuit deux adultes de la même espèce. Les tests réalisés sur $O$. tsawytscha à partir d'autres espèces d'Oncorhynchus ne faisaient pas apparaitre de réponses olfactives caractéristiques.

Les observations de terrain menées en Angleterre par SOLOMON (1973) sur différents lots de saumon atlantique relâchés au stade "smolt dans trois rivières d'estuaires voisins soutiennent également l'hypothèse phèromonale selon laquelle la présence de jeunes poissons provoque l'attraction spécifique des adultes.

D'autres observations comportementales menées aux Etats Unis sur la Truite arc-en-ciel mettent en évidence l'intervention de phéromones sexuelles. NEWCOMBE et HARTMAN (1973) montrèrent à l'aide d'un aquaolfactomètre en $Y$ (fig. 2) que l'odeur de poissons en voie de frayer présentait un fort effet attractif aussi bien sur les mâles que sur les femelles, tandis que celle provenant de poissons ne frayant pas avait peu d'effet. Utilisant un autre type d'aquaolfactomètre, dénommé * bassin optomoteur * (fig. 3) EMANUEL et DODSON (1979) ont montré que les truites mâles matures étaient attirées par l'odeur de femelles en voie de frayer et que le liquide ovarien constituait le stimulus olfactif contrôlant l'orientation et la cinétique de la réaction rhéotropique de ces poissons expérimentaux. 


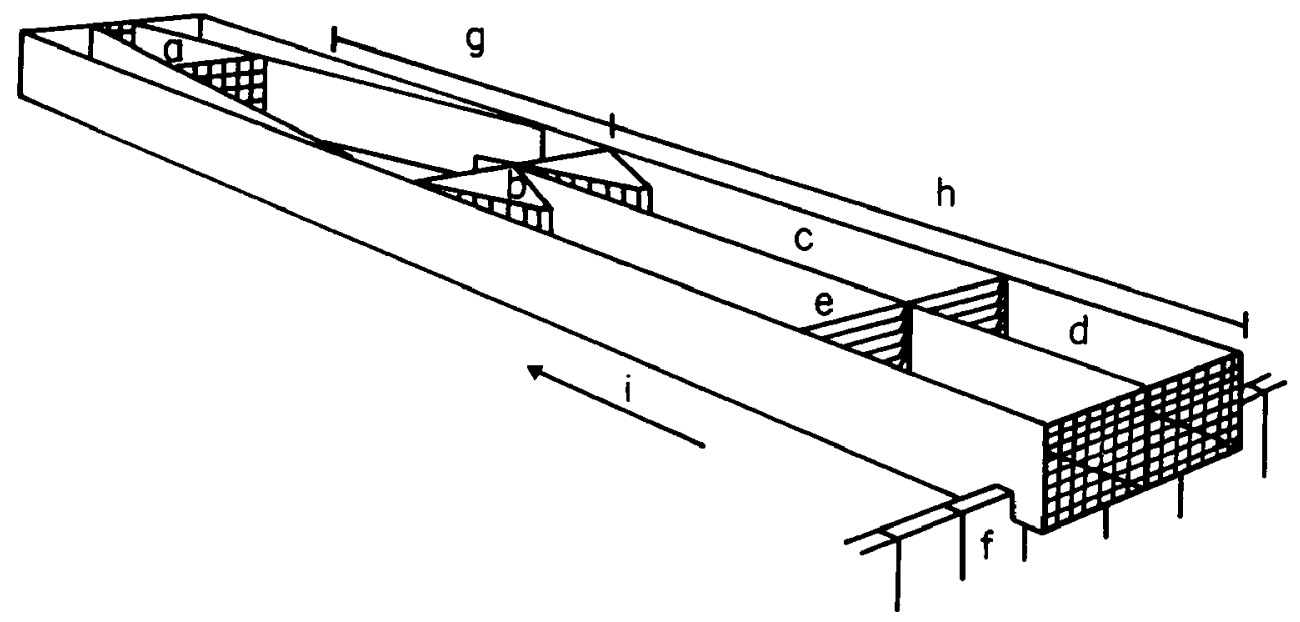

Figure 2 : Olfactomètre en $Y$.
a : compartiment de départ
b : bouche d'accès
$c$ : chenal
d : compartiment réservé à l'attractif éventuel
e : volet déflecteur
$f$ : support
g : partie de l'olfactomètre recouverte d'un panneau translucide
h : partie de l'olfactomètre recouverte d'un panneau opaque
i : direction du courant d'eau

Dimensions de l'olfactomètre : $\mathrm{L}: \mathbf{6 , 2} \mathrm{m} ; \mathrm{I}: \mathbf{0 , 6} \mathrm{m}$; Prof. 0,3 m (d'après NEWCOMBE et HARTMAN, 1973). 


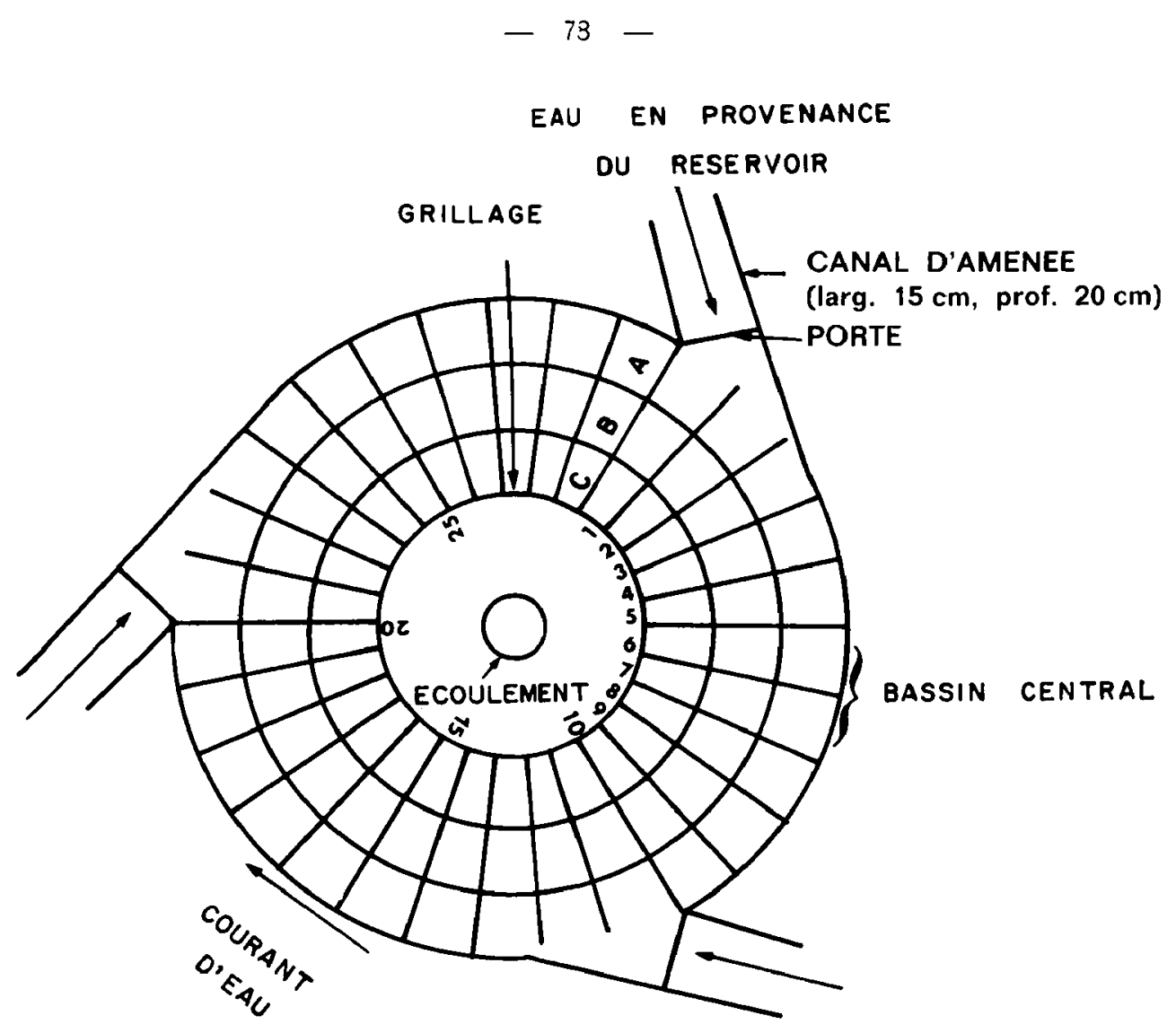

Figure 3 : "Bassin optomoteur" permettant d'observer les réponses d'orientation et la cinétique de la réaction rhéotropicjue de truites mâles soumises à des extraits ovariens (d'après EMANUEL et DODSON, 1978).

NORDENG (1977), ayanı étudié les mouvements et périodes migratoires des trois espèces de Salmonidés (S. alpinus, $S$. trutta et $S$. salar) presentes dans une rivière du Nord de la Norvège ("Salangen river system "), propose une hypothèse phéromonale susceptible de résoudre le problème posé par le mécanisme du "homing" dans son ensemble.

L'observation de populations appartenant à ces trois espèces lui permet d'effectuer deux constatations essentielles :

1) les "smolts" ont commencé leur migration aval quelque temps avant que les premiers adultes de la même espèce arrivent à l'embouchure ;

2) les mouvements de migration aval des smolts et de migiation amont des adultes se superposent pendant une période spécifique et un "trafic à deux voies" s'instaure dans la rivière et dans les eaux côtières. Considérant ces faits, NORDENG propose d'expliquer la remontée des adultes par l'attraction phéromonale exercée par les smolts descendant au même moment en mer, tandis que l'orientation finale vers les sites de fraie pourrait être déterminée par la présence de phéromones venant d'une part des populations de jeunes ( parr ") en place et, d'autre part, des premiers adultes arrivés. 


\section{C - CONCLUSION}

II apparait donc au terme de cette brève revue que la chémoréception et plus précisément la reconnaissance olfactive intraspécifique jouent un rôle déterminant dans la remontée migratoire des différentes espèces de Salmonidés anadromes ètudiées.

L'origine histophysiologique des phéromones modulant la migration reproductrice apparait double : épidermique (mucus) et gonadique (produits sexuels).

Tenant compte des récentes observations de NORDENG (1977) et des expériences de NEWCOMBE et HARTMAN (1973) et de EMANUEL et DODSON (1979) il semble fort probable que les phéromones d'origine épidermique ( $v u$ certaines propriètés caractéristiques du mucus : stabilité physico-chimique, activité à dose très faible et spécificité d'action), contribuent au regroupement des poissons avant la fraie, au choix et à la remontée de l'affluent spécifique, tandis que l'action des phéromones sexuelles induirait (vraisemblablement en synergie) l'attraction en masse au niveau des sites de fraie et le comportement sexuel des reproducteurs.

Cette hypothèse phéromonale à support olfactif $r$ 'exclut nullement d'autres hypothèses accordant une importance soit à des facteurs intrinseques (contrôle génétique de la migration; voir à ce sujet BRANNON,1967 et RALLEIGH, 1967). soit à d'autres voies sensorielles telles que la vision, ou encore à des paramètres physiques tels que la température et l'oxygénation et doit être avant tout considérée en complémentarité. Toutefois, l'emphase donnée ici aux phéromones s'accorde avec leurs réelles perspectives d'avenir. L'intérêt agro-économique évident résultant de l'utilisation de telles substances d'action spécifique (contrôle des populations de Salmonidés "sauvages " ou non : "searanching ") demeure malheureusement masqué par la complexité de leur analyse physico-chimique.

Ce fait, imputable en majeure partie aux difficultés que rencontrent actuellement la naissance et le développement de tout secteur d'études scientifiques d'importance plutôt qu'à une technicité insuffisante, est regrettable si l'on considere la part très importante réservée aux Salmonidés dans la politique aquicole suivie aujourd'hui de par le monde industrialisé (Suède. Norvège, Islande, USA, Japon. France en particulier).

\section{RESUME}

Si l'on possède encore bien peu d'informations sur les trajets suivis et sur les moyens d'orientation utilisés par les différentes espèces de Salmonidés au cours de la phase océanique de leur migration. la remontée finale des eaux proches de l'estuaire jusqu'aux sites de fraie dépend principalement du sens chimique.

Nous présentons ici un certain nombre de travaux démontrant la prééminence du rôle joué par l'olfaction et l'intervention probable de médiateurs chimiques spécifiques (ou phéromones) dans ce phénomène du "homing ". 


\section{ABSTRACT}

Although our knowledge on paths and orientation means of salmonid species during the oceanic part of their migration is limited, "homing " seems to be mainly dependent on a chemical sense.

This paper presents some data demonstrating the predominant role of olfaction and the way pheromones can function in this phenomenon.

\section{REFERENCES BIBLIOGRAPHIQUES}

BRANNON E.L., 1967. Genetic control of migrating behavior of newly emerged sockeye salmon fry. Prog. Rep., 16, 31 p.

BUCKLAND F., 1880. "Natural History of Bristish fishes". Unwin. Bros, SPCK

CARLIN B., 1959. Results of salmon smolt tagging in the Baltic area. Rapp. P. V. Reun. Cons. Int. Explor. Mer, 147, 89-96.

CARLIN B., 1968. Salmon conservation, tagging experiments, and migrations of salmon in Sweden. Atl. Salmon. Assoc. Montreal, Lect. Ser., 22 p.

CHIDESTER F.E., 1924. A critical examination of the evidence for physical and chemical influences on fish migration. J. Exp. Biol., 22, 118-144.

COLLINS G.B., 1952. Factors influencing the orientation of migrating anadromous fishes. U.S. Fish. Bull., 52, no 73, 375-396.

COOPER J.C., HASLER A.D., 1976. Electrophysiological studies of morpholineimprinted coho salmon (Oncorhynchus kisutch) and rainbow trout (Salmo gairdneri). J. Fish. Res. Bd. Can., 33, 688-694.

COOPER J.C., SCHOLZ A.T., 1976. Homing of artificially imprinted steelhead (Rainbow) trout, Salmo gairdneri. J. Fish. Res. Bd. Can., 33, 826-829.

COOPER J.C., SCHOLZ A.T., HORRALL R.M., HASLER A.D., MADISON D.M., 1976. Experimental confirmation of the olfactory hypothesis with homing artificially imprinted coho salmon (Oncorhynchus kisutch).

J. Fish. Res. Bd. Can., 33, 703-710.

CRAIGIE E.H., 1926. A preliminary experiment on the relation of the olfactory sense to the migration of the sockeye salmon (Oncorhynchus nerka Walbaum). Trans. Roy. Soc. Can., 20, 215-224.

DONALDSON R. and ALLEN G.H., 1957. Return of silver salmon Oncorhynchus kisutch (Walbaum) to point of release. Trans. Am. Fish. Soc., 87, 13-22.

DOVING K.B., ENGER P.S., NORDENG H., 1973. Electrophysiological studies ont the olfactory sense in char (Salmo alpinus L.). Comp. Biochem. Phy. siol., 45A, 21-24.

DOVING K.B., NORDENG H., OAKLEY B. 1974. Single unit discrimination of fish odours released by char (Salmo alpinus L.) populations.

Comp. Biochem. Physiol., 47A, 1051-1063.

EMANUEL M.E., DODSON J.J., 1979. Modification of the rheotropic behavior of male rainbow trout (Salmo gairdneri) by ovarian fluid.

J. Fish Res. Bd. Can., 36, 63-68 
FONTAINE M., 1975. Migration of marine and amphibaline fish. In : Adv. in Marine Biology, RUSSEL and YONGE Edts., Academy Press London New York, San Franscico, vol. 13, 241-355.

GOZ H., 1941. Uber den Art - und Individualgeruch bei Fischen. Z. Vergl. Physiol. 29, 1-45.

HARA T.J., 1974. Is morpholine an effective olfactory stimulant in fish? J. Fish Res. Bd. Can., 31, 1547-1550.

HARA T.J., MAC DONALDS S., 1975. Morpholine as olfactory stimulus in fish. Science, 187, 81-82.

HARA T.J., LAW Y.M.C., HOBDEN B.R., 1973. Comparison of the olfactory response to amino-acids in rainbow trout, brook trout, and white fish. Comp. Biochem. Physio!., 45A. 969-977.

HARDEN JONES, F.R., 1968 Fish migration. St Martin's Press, New York, 420 p.

HASLER A.D. 1956. Perception of pathways by fishes in migration. Quart. Rev. Biol., 31, 200-209.

HASLER A.D., 1966. Underwater guideposts: homing of salmon. Univ. Wisconsin Press, Madison.

HASLER A.D., 1971. Orientation and fish migration. In Fish Physiology, HOAR and RANDALL Edts., Academy Press New York and London, vol. VI, 429-510.

HASLER A.D., WISBY W.J., 1951. Discrimination of stream odors by fishes and relation to parent stream behavior. Am. Naturalist., 85, 223-238.

HOAR W.S., 1958. The evolution of migratory behavior among juvenile salmon of the genus Oncorhynchus. J. Fish Res. Bd. Can., 15, 391-428.

HOGLUND L.B., ASTRAND M., 1973. Preferences among juvenile char (Salvelinus alpinus L.) to intraspecific odours and water currents studies with the fluviarium technique. Rep. Inst. Freshwater Res. Drottningholm, 53, 21-30.

IDLER D.R., FAGERLUND U.H., MAYO H., 1956. Olfactory perception in migrating salmon. I - L. serine, a salmon repellent in mammalian skin. J. Gen. Physiol., 39, 889-892.

JENSEN A.L., DUNCAN R.N., 1971. Homing of transplanted coho salmon. Prog. Fish Cult., 33, 216-218.

JONES J.W., 1959. "The salmon". Harper New York.

KYLE H.M., 1926. "The biology of fishes" Sidgwich and Jackson, London.

MADISON D.M., SCHOLZ A.T., COOPER J.C., HORRALL R.M., HASLER A.D., DIZON A.E. 1973. I - Olfactory hypotheses and saimon migration; A synopsis of recent findings. Fish Res. Bd. Can. Tech., Rep. 414, $35 \mathrm{p}$

NEWCOMBE C., HARTMAN G., 1973. Some chemical signals in the spawning behavior of rainbow trout (Salmo gairdneri). J. Fish Res. Bd. Can., 30, 995-997.

NORDENG $H$, 1977. A pheromone hypothesis for homeward migration in anadromous salmonids. Oikos, 28, 155-159.

NORTHCOTE T.G., 1962. Migratory behavior of juvenile rainbow trout. Salmo gairdneri, in outlet and inlet streams of Loon lake, British Columbia. J. Fish Res. Bd. Can., 19, 201-270.

OSHIMA K., HAHN W.E., GORBMAN A., 1969. Olfactory discrimination of natural waters by salmon. J. Fish Res. Bd. Can., 26, 2111-2121. 
PECK J.W., 1970. Straying and reproduction of coho salmon Oncorhynchus kisutch, planted in a lake superior tributary. Trans. Am. Fish Soc., 99, 591-595.

PFEIFFER W., 1962. The fright reaction of fish. Biol. Rev., 37, 495-511.

POWERS E.B., 1939. Chemical factors affecting the migratory movements of the Pacific salmon. Am. Assoc. Advanc. Sci., 8, 72-85

POWERS E.B., CLARK R.T, 1943. Further evidence on chemical factors affecting the migratory movements of fishes, especially the salmon. Ecology, 24, 109-113.

RALEIGH R.F., 1967. Genetic control in the lakeward migrations of sockeye salmon (Oncorhynchus nerka) fry. J. Fish. Res. Bd. Can., 24, 2613-2622.

RICHARDS I.S., 1974. Caudal neurosecretory system : possible role in pheromone production. J. Exp. Zool., 187, 405-408.

SCHEURING L., 1930. Die wanderungen der Fische. I et II. Ergeb. Biol., 1, 405-691; 7, 4-304.

SCHOLZ A.T., HORRALL R.M., COOPER J.C., HASLER A.D., MADISON D.M.,

POFF R.S., DALY R.I., 1975. Artificial imprinting of salmon and trout in lake Michigan. Wis. Dep. Nat. Resour. Fish Manage., Rep. 80, 46 p.

SOLOMON D.J., 1973. Evidence for pheromone influenced homing by migrating atlantic salmon Salmo salar L. Nature, 244, 231-232.

STASKO A.B., 1971. Review of field studies on fish orientation. Ann. N. Y. Acad. Sci, 188, 12-29.

SUTTERLIN A.M., SUTTERLIN N., 1971. Electrical responses of the olfactory epithelium of atlantic salmon (Salmo salar). J. Fish Res. Bd. Can., 28, 565-572.

TODD J.H., ATEMA J., BARDACH J.E., 1967. Chemical communication in social behaviour of a fish, the yellow bullhead (Ictalurus natalis). Science, 158, 672-673.

VON FRISCH K., 1941. Uber einen schreckstoff der Fischaut und seine biologische Bedeuntung. Z. Vergl. Physiol., 29, 46-145.

WARD H.B., 1921a. Some of the factors controlling the migration and spawning of the Alaska red salmon. Ecology 2, 235-354.

WARD H.B. 1921b. Some features in the migration of the sockeye salmon and their practical significance. Trans. Am. Fish. Soc., 50, 387-426.

WARD H.B., 1939. Factors controlling salmon migration.

Am. Assoc. Advance Sci., publ. 8, 60-71.

WHITE H.C. 1934. Some facts and theories concerning the Atlantic salmon. Trans. Am. Fish. Soc., 64, 360-362.

WISBY W. J., 1952. Olfactory responses of fishes related to parent stream behaviour. Ph.D. Thesis University of Wisconsin.

WREDE W.L., 1932. Versuche uber den Artduft der Elritzen. Z. Vergl. Physiol., 17, 510-519. 